BULL. AUSTRAL. MATH. SOC.

MOS 2093

VOL. $2(1970), 285-286$.

\title{
Congruences on orthodox semigroups
}

\section{J. C. Meakin}

The kernel of a congruence on a regular semigroup $S$ is the set of congruence classes which contain idempotents of $S$. The regular kernel of a congruence $\rho$ on an orthodox semigroup (a regular semigroup whose set of idempotents forms a subsemigroup) is defined to be the set of maximal regular subsemigroups of the elements of the kernel of $\rho$. It is proved that a congruence on an orthodox semigroup is uniquely determined by its regular kernel. The regular kernel of a congruence on an orthodox semigroup is characterized as a "regular kernel normal system" (by analogy with Preston's kernel normal systems of inverse semigroups) and an explicit method of constructing the congruence associated with a prescribed regular kernel normal system is provided. These results are simplified in the case of idempotent-separating congruences and inverse semigroup congruences on orthodox semigroups.

Two congruences on an orthodox semigroup $S$ are defined to be idempotent-equivalent if they induce the same partition of the set $E_{S}$ of idempotents of $S$. Such congruences are examined from the point of view of their regular kernels. Those partitions of $E_{S}$ which are induced by congruences on $S$ are characterized and the maximal and minimal congruences inducing a given partition of $E_{S}$ are determined. A construction of the regular kernels of the meet and join of two idempotent-equivalent congruences $\rho$ and $\sigma$ on $S$ (in terms of the regular kernels of $\rho$ and $\sigma$ ) is obtained.

The final chapter is concerned with one-sided congruences on inverse semigroups. These are examined from the point of view of their kernels and a theory of one-sided kernel normal systems of inverse semigroups and

Received 10 November 1969. Thesis submitted to Monash University, July 1969. Degree approved, September 1969. Supervisor: Professor G.B. Preston. 
idempotent-equivalent one-sided congruences on inverse semigroups is developed. 